\title{
Behind closed doors: systematic analysis of breast cancer consultation communication and predictors of satisfaction with communication
}

\author{
Thomas F. Hack 1,2*, Tom Pickles ${ }^{3,4}$, J. Dean Ruether ${ }^{5,6}$, Lorna Weir ${ }^{3,4}$, Barry D. Bultz ${ }^{5,6}$ and Lesley F. Degner' \\ 'Faculty of Nursing, University of Manitoba, Winnipeg, MB, Canada \\ ${ }^{2}$ CancerCare Manitoba, Winnipeg, MB, Canada \\ ${ }^{3}$ British Columbia Cancer Agency, Vancouver, BC, Canada \\ ${ }^{4}$ Faculty of Medicine, University of British Columbia, BC, Canada \\ ${ }^{5}$ Tom Baker Cancer Centre, Calgary, AB, Canada \\ ${ }^{6}$ Faculty of Medicine, University of Calgary, AB, Canada
}

* Correspondence to: Asper Clinical Research Institute/St. Boniface Research Centre, Room CR3018, 369 Taché Avenue, Winnipeg, Manitoba, Canada $\mathrm{R} 2 \mathrm{H} 2 \mathrm{~A} 6$

E-mail: thack@sbrc.ca

Received: 10 November 2008 Revised: 21 April 2009 Accepted: 24 April 2009

\begin{abstract}
Objective: The purpose of this investigation was to explicate the content of primary adjuvant treatment consultations in breast oncology and examine the predictive relationships between patient and oncologist consultation factors and patient satisfaction with communication.

Methods: The recorded consultations of 172 newly diagnosed breast cancer patients from four Canadian cancer centers were randomly drawn from a larger subset of 481 recordings and examined by three coders using the Medical Interaction Process System (MIPS); a system that categorizes the content and mode of each distinct utterance. The MIPS findings, independent observer ratings of patient and oncologist affective behavior, and derived consultation ratios of patient centeredness, patient directedness, and psychosocial focus, were used to predict patient satisfaction with communication post-consultation and at 12-weeks post-consultation.

Results: Biomedical content categories were predominant in the consultations, accounting for $88 \%$ of all utterances, followed by administrative (6\%) and psychosocial (6\%) utterances. Post-consultation satisfaction with communication was significantly higher for older patients, those with smaller primary tumors and those with longer consultations. Smaller tumor, lack of patient assertiveness during the treatment consultation and having the consultation with a radiation rather than medical oncologist were significantly predictive of greater satisfaction at 12-weeks post-consultation.

Conclusions: Adjuvant treatment consultations are characterized by a high degree of informationgiving by the physician, a predominance of biomedical discussion and relatively minimal time addressing patients' psychosocial concerns. Controlled trials are needed to further identify and address the contextual features of these consultations that enhance patient satisfaction. Copyright (C) 2009 John Wiley \& Sons, Ltd.
\end{abstract}

Keywords: cancer; oncology; consultation; communication; satisfaction

\section{Introduction}

Optimal medical management during the first few months following a diagnosis of breast cancer includes full discussions about disease status, prognosis and the treatment plan. The primary adjuvant treatment consultation is of particular importance to women with breast cancer; an occasion when the oncologist outlines adjuvant treatment options so that patients may make informed treatment decisions. One challenge for communication research in oncology is to operationally define and measure indicators of patientprofessional communication [1]. Several measurement systems have been developed for this purpose, and despite not being contradictory or exclusive, these measurement systems lack common ground. A meta-analysis [2] and review [3] of these measurement systems suggest five common categories of patient-physician communication: (a) information giving, (b) question asking, (c) partnership building, (d) rapport building, and (e) emotional support.

Despite these communication measurement systems, communication research in oncology is limited by a lack of theoretical models to explain oncology communication. In response to this deficiency, two new conceptual frameworks have recently been developed [4-5]. Both frameworks are useful in identifying variables that shape 
communication exchange in the patient-professional dyad, and communication goals have a central place in each model. The Comskil Model [4], developed as an aid to communication skills enhancement, identifies 5 core components: communication goals, strategies, skills, process tasks, and cognitive appraisals. The model by FeldmanStewart et al. [5] is broader in scope, affording the provider and patient equal prominence, and regarding the external environment as having an important influence on the attributes of each participant in the consultation. The 4 core components of this model include: communication goals, participant attributes, process of communication, and environment.

In both conceptual frameworks, the relationships between the components of the models and patient and provider outcomes are implied but not explicitly stated. What is lacking in the empirical literature are summative, theoretical accounts of the relationships between consultation factors and patient outcomes [6]. Toward this aim, there is a need to systematically document the relationships between elements of communication exchange and patient-reported outcomes [1]. To date, the most common patient-reported outcomes to be assessed include understanding, satisfaction and well-being [7], with patient satisfaction being the most widely used $[6,8]$.

Patient satisfaction and well-being have been shown to be positively related to several communication aspects of the cancer consultation, including having patients' questions and concerns answered during the consultation [9-10], and physicians who score high on patient ratings of their caring behaviors and information giving [11]. Patient satisfaction following the initial cancer consultation is predicted by the affective quality of the oncologist's communication style as well as the communication style of the patient [12]. Oncologist variables found to be significantly related to patient satisfaction include friendliness and anger (at 1-week post-consultation), and social behavior and information giving (at 3-months post-consultation). Significant patient characteristics include negative talk, interest, and dominance (at 1-week post-consultation), and negative talk, directions, and anger (at 3-months post-consultation).

Communication problems reported by women with breast cancer prior to adjuvant treatment have been found to be significantly related to psychological well-being three months later [13]. The most commonly reported communication problems were difficulty in understanding physicians (reported by $50 \%$ of patients), difficulty in expressing feelings $(46 \%)$, desire for more control $(45 \%)$, and difficulty asking questions of physicians (43\%). In a sample of 454 oncology outpatients, well-being was significantly higher when patients perceived that their physicians were highly attentive and empathic [14].

It is difficult to synthesize the findings from studies that have measured patient satisfaction with communication as the primary outcome given the diversity in the way patient satisfaction has been conceptualized, differences in sample characteristics across studies, and the plethora of patient satisfaction questionnaires in use. Patient satisfaction instruments are also known for ceiling effects [15]. While many arguments have been put forward to explain these ceiling effects, patient satisfaction with communication remains an important variable that is significantly related to psychological morbidity of patients [16].

Although various communication measurement systems exist, most studies that have used these measurement tools have reported on a subset of consultation factors. To our knowledge a 'complete set' or comprehensive account of the primary treatment consultation in oncology has not been reported. The primary purpose of this study was to conduct a complete, systematic explication of a representative subset of these consultations with respect to the content and mode of all patient and physician utterances. The secondary purpose of this study was to determine whether consultation features are predictive of patient satisfaction with communication immediately post-consultation and at 12-weeks post-consultation. Given the growing empirical voice promoting the merits of shared decision making in oncology, we hypothesized that consultations characterized by patient-centeredness, a psychosocial focus, and patient-directedness would generate significantly higher levels of patient satisfaction with communication than consultations lacking these descriptive characteristics. Moving beyond consultation characteristics in accordance with our conceptual frameworks, we were also interested in whether patient (age, education, urban vs rural residence, surgical procedure, tumor size, and adjuvant therapy type) and oncologist (practice specialty) characteristics might influence patient satisfaction with communication.

\section{Methods}

\section{Patient sample}

Eligible patients included women with breast cancer who presented to a tertiary or community oncology clinic for their primary adjuvant treatment consultation, and who previously participated in a study of the benefits of providing women with breast cancer with an audio-recording of their primary adjuvant treatment consultation [17]. All patients were older than 18 years of age and discerned to be free of any cognitive impairment or 
other limiting factor precluding their ability to provide informed consent. Patients were accrued from six cancer treatment facilities in four Canadian cities, and data analyses were run on a final sample of 172 patients. These patients were recruited from the clinics of 19 medical and 18 radiation oncologists at the British Columbia Cancer Agency, Vancouver, Canada $(n=12)$, the Tom Baker Cancer Centre, Calgary, Canada $(n=9)$, CancerCare Manitoba, Winnipeg, Canada $(n=6)$, and the Cross Cancer Institute, Edmonton, Canada $(n=10)$.

\section{Study design and procedure}

The study protocol was approved by the review committees for ethics in human subjects research at each institution, and all patients and oncologists provided informed, written consent to participate.

Prior to the treatment consultation, the clinical research nurse obtained informed consent and administered a patient sociodemographic and illness profile. The clinical research nurse met each patient after the consultation to administer the patient satisfaction with communication measure and furnish assigned patients with their consultation recordings.

A copy of this scale was given to the patient in a sealed envelope with an instruction to keep the envelope sealed until contacted by the research nurse approximately 12 weeks later, at which time the nurse phoned the patient to record the answers to the questionnaire items as provided by the patient via telephone.

\section{Audiotape sample}

Stratified random sampling was used to select the audiotapes to be coded. The recordings were stratified on the basis of oncologist to ensure the oncologists had statistically equivalent representation in the set of coded recordings. To account for the possibility that the practice style of oncologists might change with repeated tapings of consultations, the audiotapes were organized chronologically by date of interview prior to random sampling using random number generating software. To obtain a representative sample of the available recordings at the goal rate of one-third, $240(50 \%)$ of the 481 available audiotapes were identified to be coded. This accounted for reductions in the number of audiotapes excluded as a result of low inter-rater reliability scores $(n=17)$, such poor quality as to preclude complete analysis $(n=29)$, patient attrition prior to follow-up $(n=5)$, improper randomization $(n=1)$, a non-primary, recurrent cancer $(n=1)$, a missing audiotape $(n=1)$, a consultation with three physicians $(n=1)$, and one consultation led by a nononcologist $(n=1)$. After the representative sample of recordings had been generated, a secondary convenience sample of 12 of the remaining audiotapes was generated for use in preliminary training. The 172 coded audiotapes in the final sample constituted $35.8 \%$ of the available recordings.

\section{MIPS}

The Medical Interaction Process System (MIPS) [18] is an objective, reliable, and valid instrument for analysing oncology consultations. The MIPS was developed by combining categories adapted from the Roter Interaction Analysis System (RIAS) with those derived from detailed examination of audiotapes [19] and videotapes [20] of oncology consultations. The MIPS classifies patient-physician exchanges in terms of 'content' and 'mode' of exchange. The basic coding unit is an utterance. A separate utterance is coded for each independent and every non-restrictive dependent clause of a sentence. For example, 'You need to have a scan because your cancer may have progressed' consists of two utterances.

Each unit of utterance is assigned one 'content' code and one 'mode' of exchange, and may be initiated by either the patient or oncologist. 'Content' categories (see Table 1) reflect the topics being addressed in each utterance. 'Modes' (see Table 1) refer to the process or function of an utterance, e.g. 'giving information' or 'asking questions'. A coding example follows:

(1) Oncologist: 'Do you have pain in the breast?' (2) Patient: 'It hurts below the incision line.'

\begin{tabular}{llcl}
\hline Unit & Speaker & Content category & Model of exchange \\
\hline$(1)$ & Oncologist & Medical & Asks closed question \\
$(2)$ & Patient & Medical & Gives information \\
\hline
\end{tabular}

In addition to coding the content and mode of each utterance, there are seven 'global affective' categories that are rater scored on a continuous scale from $0-10$. Four of these categories pertain to oncologist behavior: level of patient centeredness, friendliness, sensitivity, and nervousness. The remaining three categories are for patient behavior: level of anxiety, hostility, and nervousness.

The current research team elected to use the MIPS in the analyses of audiotapes given its relative advantages. First, the MIPS categories are sufficiently detailed to capture the diversity and complexity of information exchanged during a cancer consultation. Second, the MIPS allows for the application of more than one code for a single mode utterance. Third, the nature of affective responses can be coded, e.g. an utterance may be coded as 'provides reassurance', and additional information can be noted regarding the type of reassurance, i.e. genuine or false [18]. 
Table I. MIPS content and mode categories

\begin{tabular}{|c|c|}
\hline \multicolumn{2}{|l|}{$\overline{\text { Content categories }}$} \\
\hline Intro & Introductory greetings \\
\hline Medical & $\begin{array}{l}\text { All medical details pertaining to the } \\
\text { present consultation }\end{array}$ \\
\hline Omedical & Other medical matters \\
\hline Tests & Past and future test results \\
\hline Tmt & Main cancer treatment \\
\hline S.effs & Side effects of main treatment \\
\hline Drugs & $\begin{array}{l}\text { Treatments and drugs prescribed to } \\
\text { prevent/alleviate side effects caused by } \\
\text { treatment }\end{array}$ \\
\hline Ps/Med & $\begin{array}{l}\text { A statement, question, or concern that } \\
\text { could relate equally to psychological } \\
\text { feelings or physical (medical) symptoms }\end{array}$ \\
\hline Psych & $\begin{array}{l}\text { Psychological (patient's feelings and } \\
\text { emotions) }\end{array}$ \\
\hline L.style & $\begin{array}{l}\text { Health and lifestyle issues such as diet, } \\
\text { drug habits, complementary therapies, } \\
\text { and leisure pursuits }\end{array}$ \\
\hline Soc/Dem & Social/demographic characteristics \\
\hline Soc/Con & $\begin{array}{l}\text { Social/personal conversation not directly } \\
\text { connected with the consultation }\end{array}$ \\
\hline Ad/Prac & Administrative practical details \\
\hline End & Goodbyes, closing statements \\
\hline Uncoded & $\begin{array}{l}\text { Utterances that lack the necessary } \\
\text { information to code them }\end{array}$ \\
\hline \multicolumn{2}{|l|}{ Mode categories } \\
\hline Asks questions & Expresses \\
\hline I. Open question ${ }^{\mathrm{a}}$ & I. Irritation ${ }^{\mathrm{b}}$ \\
\hline 2. Closed question & 2. Gratitude ${ }^{b}$ \\
\hline 3. Leading question ${ }^{\mathrm{a}}$ & 3. Apology ${ }^{b}$ \\
\hline 4. Multiple question ${ }^{\mathrm{a}}$ & 4. Empathy/Psychological support ${ }^{\text {ab }}$ \\
\hline 5. Focused open question ${ }^{\mathrm{a}}$ & 5. Laughter ${ }^{\mathrm{b}}$ \\
\hline Checks & 6. Positive response ${ }^{b}$ \\
\hline I. Information & 7. Negative response ${ }^{b}$ \\
\hline 2. Understanding & Agreement \\
\hline 3. Summarises ${ }^{a}$ & Registers information \\
\hline Gives & Facilitates speech ${ }^{a}$ \\
\hline I. Information (neutral) & Interrupts \\
\hline 2. Information (positive) & Asks for repetition \\
\hline 3. Information (negative) ${ }^{b}$ & Seeks information \\
\hline 4. Reassurance ${ }^{\text {ab }}$ & Directs/Advises \\
\hline $\begin{array}{l}\text { 5. False/Premature } \\
\text { assurance }^{\mathrm{ab}}\end{array}$ & Requests/Preference ${ }^{c}$ \\
\hline Orientation $^{\mathrm{a}}$ & Inappropriate behavior ${ }^{\mathrm{a}}$ \\
\hline
\end{tabular}

${ }^{\mathrm{a} S}$ Pecific to oncologist.

${ }^{\mathrm{b}}$ Affective categories.

'Specific to patient.

\section{MIPS training and reliability}

The principal investigator and one of the three raters were trained by the developer of the MIPS, Dr Sarah Ford, using the MIPS training manual. During the preliminary training period, inter-rater reliabilities were assessed on each of 12 coded consultations, and training continued until the reliability estimate was above 0.80 for all content and mode categories, and above 0.95 for the total number of patient and oncologist utterances.

The randomized audiotapes were further stratified by data collection site and randomly allocated, using random number generating software, among the three raters. Two raters coded 50 of the consultation recordings, while the third rater coded 72 of them. Inter-rater reliability checks were performed after approximately every 4 or 5 coded consultations.

While the MIPS allows for patients' verbal communication signals to the oncologist - cues/concerns and oncologist expressions of patient concerns to be coded, cues and concerns were not formally coded for the present study. Rather, a mode entitled 'inappropriate behavior' was added to the coding scheme, defined as notable oncologist speech improprieties or glaring speech omissions that occured either independently of, or in association with, patient speech.

\section{Patient satisfaction with communication}

Patient satisfaction with communication was measured using the Patient Perception Scale [21]; a 9-item patient report measure of patient-centered communication with the oncologist during the consultation. This scale, reduced from the originally validated 14 -item [22-23] and 12-item versions [24], includes items that assess the degree to which the patient feels the oncologist has adequately addressed the patient's disease concerns and been patient-focused during the consultation (e.g. How satisfied were you with the discussion of your problem? To what extent did the doctor listen to what you had to say? To what extent did the doctor explain treatment? How well do you think your doctor understood you today?). Each item is rated on a 4-point scale, and the possible range of scores is 9-36, with 9 representing the highest possible patient satisfaction, i.e. report of patient-centered communication. Patient-centered communication is operationally defined as (a) eliciting and understanding the patient's perspective, (b) understanding the patients psychosocial context, (c) establishing a shared understanding of the problem and treatment in accordance with the patient's values, and (d) involving the patient in making choices to the degree desired by the patient [25]. These four features are captured by the Patient Perception Scale. Given our additional interest in correlating the three consultation features of patient-centeredness, psychosocial focus, and patient-directedness with our outcome measure, the Patient Perception Scale was well suited for this study. This measure has been shown to be predictive of patient health status and efficiency of care (reduced diagnostic tests and referrals) [24]. Cronbach's coefficient alpha for this measure in the present study was 0.88 .

\section{Data analyses}

Unix SAS V 9.1 software was used for all analyses; descriptive statistics and correlation matrices were 
generated for all measures. Almost all of the consultations consisted of three parts: (a) review of general medical history (e.g. previous illnesses, prior hospitalizations, family history of heart problems, etc.); (b) physical examination; and (c) discussion of the patient's cancer, i.e. explanation of the disease and treatment. Given our primary interest in the latter and out of concern for patient privacy when listening to the recorded consultation alone or with others, any recording of the physicial examination (approx. $5 \mathrm{~min}$ ) or general systems history (approx. $10 \mathrm{~min}$ ) portion of the consultation was discouraged and omitted from analysis if present on the audiotape.

The presence of a third individual in the consultation, i.e. a family member, spouse, partner, or significant aquaintance of the patient, was noted in $106(61.6 \%)$ of the 172 consultations, for a total of 2392 utterances representing $3.0 \%$ of all utterances. These utterances were not included in the statistical analyses given our interest in the patient-oncologist dyad, our assumption that the utterances of the accompanying individual are not valid proxy responses for the patient, and the relatively infrequent speech of the companions despite their presence in close to two-thirds of the consultations. There were 14 consultations $(8 \%)$ that included utterances of two oncologists. For these 2-doctor consultations, the 4050 utterances $(5.0 \%$ of all utterances) of the non-primary oncologist were not included in the analyses. There were also a few consultations for which the audiotaping equipment was turned on after the introductory greetings or prior to departing words of farewell. To maintain consistency across consultations, the 169 introductory utterances $(0.2 \%$ of all utterances) and 227 farewell utterances $(0.3 \%$ of all utterances) were excluded from the analyses.

Multivariate tests were run to examine the relationships between consultation factors and the dependent measure (patient satisfaction with communication). The control variables included patient age, education, residence (urban/rural), surgical procedure, tumor size, and adjuvant therapy type. The predictor variables included the duration of the consultation in minutes, the practice specialty of the oncologist (medical oncologist/radiation oncologist), whether or not the patient received a consultation recording, the seven 'global affective' rater categories, and three consultation ratios (patient directedness, patient centeredness, and psychosocial focus) computed by formulas driven by content and mode combinations. These three ratios (see Table 2) were developed in consultation with Dr Ford to capture groupings of content and mode combinations that reflect the current empirical literature on each of the three ratio topics (the larger number of content and mode categories precluded a cluster analysis of the consultation data). Multicollinearity was a potential concern given the possible commonality of content and mode categories used to compute each of the ratios, but no analytic adjustment was required because the highest of the inter-ratio correlations was 0.21 . For all significant $(p<0.05)$ regression models, only those significant $(p<0.05)$ predictors for which Pillai's trace statistic was also significant $(p<0.10)$ are reported.

\section{Results}

\section{Explicating the consultation}

The demographic and treatment information for this sample is provided in Table 3 . The average duration of the primary adjuvant treatment consultation (excluding the physical examination and historical review of general systems) was $23.0 \mathrm{~min}$ $(\mathrm{SD}=13.4 \mathrm{~min})$. The total number of utterances for the 172 consultations was 73403 (Patient19625 utterances (26.7\%); Oncologist-53778 $(73.3 \%))$. A breakdown of these consultations by content and mode is presented in Table 4, according to the percentage of utterances realized for each category. Biomedical content categories were predominant in the consultations, accounting for $88.3 \%$ of all utterances, followed by administrative $(5.9 \%)$ and psychosocial $(5.8 \%)$ utterances. It was most common for oncologists to lead the consultations and for the patients to follow along in the same content category; therefore, the percentages of content utterances per category are, not surprisingly, similar for oncologists and patients.

With respect to mode categories, the most frequent oncologist mode was 'gives information' to the patient; an average of 222.9 utterances per consultation $(71.3 \%$ of the total utterances of the oncologist). The second and third most common modes for the oncologist were 'directs/advises' (20.6 utterances per consult; $6.6 \%$ of oncologist utterances), followed by 'registers information' (15.2 utterances per consult; $4.9 \%$ of oncologist utterances). The most common mode for patients was 'registers information' (46.8 utterances per consult; $41 \%$ of patient utterances), followed by 'gives information' (39.7 utterances per consult; $34.8 \%$ of patient utterances). The average number of question utterances per consultation was 6.7 for the patient and 10.3 for the oncologist (a question typically consists of 1-2 utterances, so the true number of questions asked by patients and oncologists is less than the mean utterance count). Of the 10.3 average question utterances of the oncologist, a little more than half of these utterances (5.8) represented closed questions (a question that requires a yes/no response). Open questions (questions that encourage patients to respond more fully) were rare among oncologists; an average of 0.7 utterances per consultation. The 

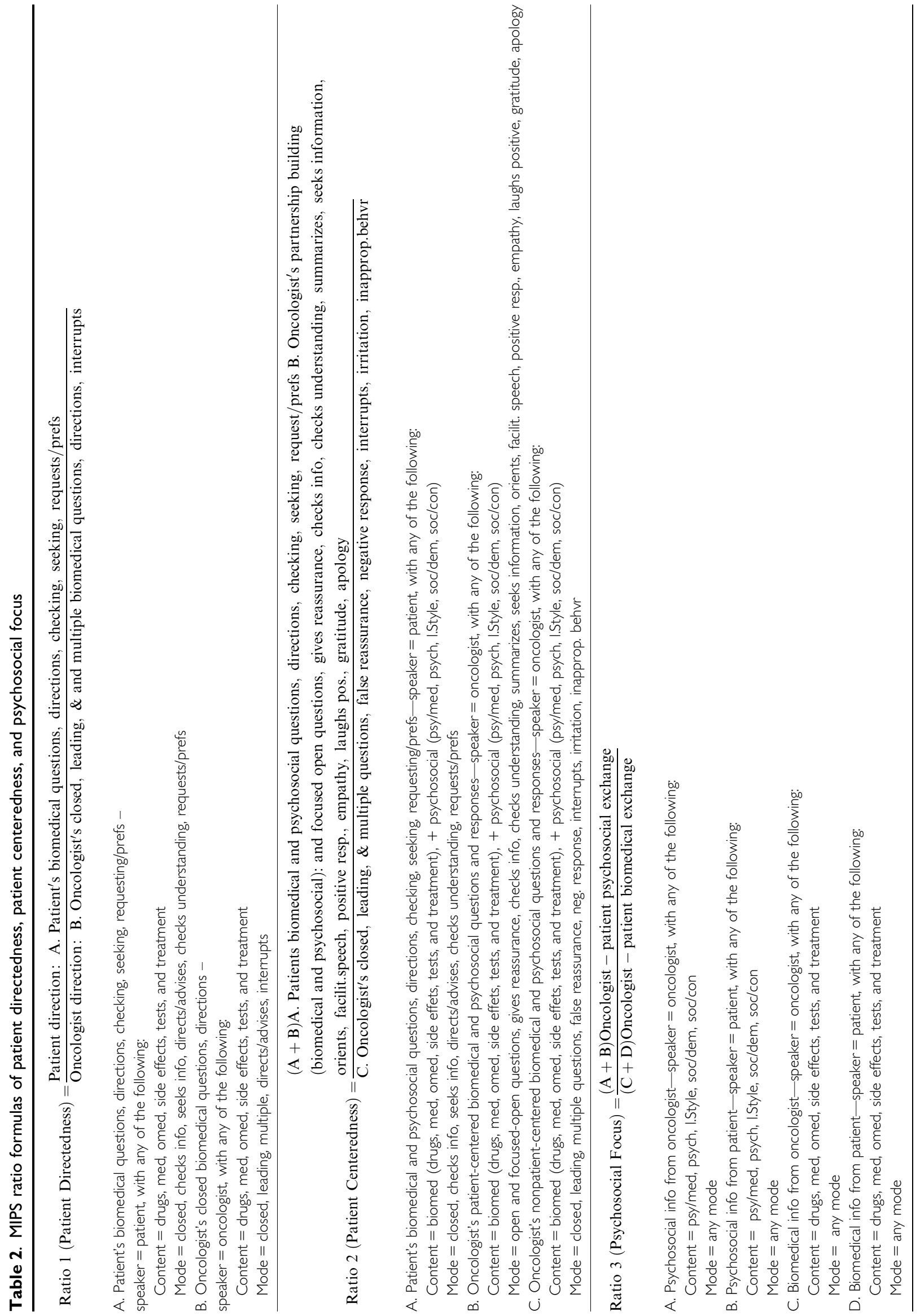
Table 3. Patient demographic and treatment information $(n=172)$

\begin{tabular}{lc}
\hline Variable & No. of patients (\%) \\
\hline Age, years & \\
(Mean $=56.0$, SD $=12.9)$ & \\
Education & $23(13.4)$ \\
$\leqslant$ Grade I0 & $59(34.3)$ \\
Grades II-I3 & $90(52.3)$ \\
> High school & \\
Residence & $120(69.8)$ \\
Urban & $52(30.2)$ \\
Rural & \\
Surgical treatment & $100(58.1)$ \\
Lumpectomy & $50(29.1)$ \\
Modified radical mastectomy & $7(4.1)$ \\
Both treatments & $15(8.7)$ \\
Not reported/missing & \\
Tumor size, cm & \\
(Mean = 2.2, SD = I.9) & \\
Adjuvant therapy & \\
Chemotherapy & $60(38.4)$ \\
Hormone therapy & $63(36.6)$ \\
Radiation therapy & $70(40.7)$ \\
Oncologist specialty & \\
Medical oncology & \\
Radiation oncology & \\
Consultation recording receipt & \\
Yes & \\
No & \\
\hline
\end{tabular}

average number of utterances per consult for patients to check for information was 4.2, while oncologists checked for information or patient understanding an average of 8.5 utterances per consultation. Of the average number of oncologist utterances to check for understanding, 0.3 utterances were checks for thorough understanding while 4.2 utterances were cursory checks for understanding. Inappropriate responses by the oncologist were uncommon (1.6 utterances per consult), as were empathic responses by the oncologist (3.3 utterances per consult).

\section{Predictors of patient satisfaction}

The sample means and standard deviations for the patient satisfaction variables, global affective ratings, and MIPS consultation ratios are shown in Table 5 and the results of the regression analyses are shown in Table 6 (to maintain a reasonable table size, only statistically significant variables are presented in this table). Patient satisfaction with communication was high, both immediately postconsultation $(M=12.1, \quad \mathrm{SD}=3.2 ;$ range $=9-23)$ and 12 weeks later $(M=14.7, \quad \mathrm{SD}=4.6$; range $=9-30$ ).

Patient satisfaction with communication measured just a few minutes after the primary adjuvant treatment consultation was significantly predicted by patient age, breast tumor size, and the duration of the consultation: older patients, those with smaller tumors, and those with longer consultations reported the highest levels of satisfaction. Satisfaction with communication 12 weeks later was significantly predicted by tumor size, the patient assertiveness global affective rating and by medical specialty: patients with smaller tumors, those who were deemed less assertive during the consultation, and those whose primary consultation was with a radiation oncologist rather than a medical oncologist were most satisfied. None of the consultation ratios of patient-directedness, patientcenteredness, and psychosocial focus were statistically significant predictors.

\section{Discussion}

The findings show that primary adjuvant treatment consultations in breast oncology are characterized by a high degree of biomedical information given to the patient by the oncologist. This is not surprising, given the complexity of the disease trajectories and treatment regimens that are discussed during these consultations. More striking in these results is the relatively small amount of discussion time dedicated to psychosocial matters. The low ratio of psychosocial conversation to disease and treatment dialogue is similar to that reported elsewhere $[19,26,27]$, as was the low percentage $(1.5 \%)$ of time that the oncologists dedicated to checking patient understanding $[26,28]$. The determination of the optimal percentage or amount of oncologist time to be dedicated to psychosocial matters is complicated by the fact that patient satisfaction with communication is influenced by both the quality and quantity of communication exchange: the fewest of choice words spoken to the patient at an opportune moment may result in greater satisfaction than a lengthy discourse that lacks meaning to the patient.

It is not possible to answer the question 'Are oncologists communicating well with their patients?' from the study findings. Despite the infrequency of psychosocial discussion and low number of empathic utterances by the oncologists, the number of utterances deemed 'inappropriate' was also low, and the level of patient satisfaction with communication was generally high. Furthermore, it is possible that some oncologists with excellent communication skills may, with favorable consequences for the patient, defer the bulk of psychosocial discussions to the primary nurse or to a dedicated psychosocial clinician.

The findings show that satisfaction with communication immediately following the primary adjuvant treatment consultation is greatest for older patients and those with smaller tumors and longer consultations. The finding of greater satisfaction with communication among older patients 
Table 4. Primary adjuvant treatment consultation explicated by content and mode (\% of total utterances)

\begin{tabular}{|c|c|c|c|}
\hline & Patient & Oncologist & Total \\
\hline \multicolumn{4}{|l|}{ Content category } \\
\hline Biomedical & $84.2 \%$ & $89.9 \%$ & $88.3 \%$ \\
\hline Medical-cancer & 24.2 & 23.1 & 23.3 \\
\hline Cancer treatment & 32.1 & 38.9 & 37.1 \\
\hline Cancer side effects & 12.7 & 18.0 & 16.6 \\
\hline Cancer tests & 3.1 & 3.1 & 3.1 \\
\hline Cancer drugs & 1.8 & 1.7 & 1.7 \\
\hline Medical non-cancer & 9.7 & 4.5 & 5.9 \\
\hline Psych/medical symptoms & 0.7 & 0.6 & 0.6 \\
\hline Psychosocial & $8.8 \%$ & $4.6 \%$ & $5.8 \%$ \\
\hline Psychological & 2.6 & 1.4 & 1.7 \\
\hline Lifestyle/Health & 2.3 & 1.5 & 1.7 \\
\hline Sociodemographic & 3.0 & 1.3 & 1.8 \\
\hline Social conversation & 0.9 & 0.5 & 0.6 \\
\hline Administrative detail & $7.0 \%$ & $5.5 \%$ & $5.9 \%$ \\
\hline \multicolumn{4}{|l|}{ Mode category } \\
\hline \multicolumn{4}{|l|}{ Asks questions } \\
\hline I. Open question & $\mathrm{n} / \mathrm{a} \%$ & $0.2 \%$ & $0.2 \%$ \\
\hline 2. Closed question & 5.9 & 1.9 & 2.9 \\
\hline 3. Leading question & $\mathrm{n} / \mathrm{a}$ & 0.4 & 0.3 \\
\hline 4. Multiple question & $\mathrm{n} / \mathrm{a}$ & 0.1 & 0.1 \\
\hline 5. Focused open question & $\mathrm{n} / \mathrm{a}$ & 0.7 & 0.5 \\
\hline \multicolumn{4}{|l|}{ Checks } \\
\hline I. Information & 3.7 & 1.3 & 1.9 \\
\hline 2. Understanding & 0.0 & 1.5 & I.1 \\
\hline 3. Summarises & $\mathrm{n} / \mathrm{a}$ & 0.9 & 0.7 \\
\hline \multicolumn{4}{|l|}{ Gives } \\
\hline I. Information (neutral) & 34.8 & 71.3 & 61.5 \\
\hline 2. Information (positive) & 0.02 & 0.6 & 0.4 \\
\hline 3. Information (negative) & 0.04 & 0.2 & 0.2 \\
\hline 4. Reassurance & $\mathrm{n} / \mathrm{a}$ & 2.2 & 1.6 \\
\hline 5. False/premature assurance & $\mathrm{n} / \mathrm{a}$ & 0.1 & 0.1 \\
\hline Orientation & $\mathrm{n} / \mathrm{a}$ & 2.7 & 2.0 \\
\hline Registers information & 41.0 & 4.8 & 14.5 \\
\hline \multicolumn{4}{|l|}{ Expresses } \\
\hline I. Irritation & $0.03 \%$ & $0.0 \%$ & $0.01 \%$ \\
\hline 2. Gratitude & 0.3 & 0.1 & 0.1 \\
\hline 3. Apology & 0.1 & 0.2 & 0.1 \\
\hline 4. Empathy/Psychological support & $\mathrm{n} / \mathrm{a}$ & I.I & 0.8 \\
\hline 5. Laughter (positive) & 3.1 & 0.5 & 1.2 \\
\hline 6. Laughter (negative) & 0.1 & 0.0 & 0.03 \\
\hline 7. Positive response & 0.7 & 0.2 & 0.3 \\
\hline 8. Negative response & 0.6 & 0.02 & 0.2 \\
\hline Asks for repetition & 0.04 & 0.03 & 0.04 \\
\hline Requests/Preference & 0.03 & $\mathrm{n} / \mathrm{a}$ & 0.01 \\
\hline Interrupts & 0.1 & 0.2 & 0.2 \\
\hline Agreement & 4.8 & 1.1 & 2.1 \\
\hline Facilitates speech & $\mathrm{n} / \mathrm{a}$ & 0.1 & 0.1 \\
\hline Directs/Advises & 1.5 & 6.6 & 5.2 \\
\hline Seeks information & 3.2 & 0.5 & 1.3 \\
\hline Inappropriate behavior & $\mathrm{n} / \mathrm{a}$ & 0.5 & 0.4 \\
\hline
\end{tabular}

is consistent with earlier reports of breast cancer patients [16]. There were marginally significant findings of greater satisfaction with communication immediately after the consultation among patients seeing radiation oncologists for this consultation and for those who received an audio-recording of this consultation. It is noteworthy that there was a statistically significant correlation $(0.285$, $p<0.0001)$ between medical specialty and tumor size-radiation oncologists tended to see patients with smaller primary tumors, many of whom did not also receive chemotherapy. Anxiety-provoking discussion of chemotherapy was therefore less prominent in consultations with radiation oncologists compared with those with medical oncologists. Receiving a consultation recording may have 
reduced patient anxiety and otherwise enhanced satisfaction with communication, especially for patients who learned that they would not have to endure chemotherapy and its side effects. The fact that seeing a radiation oncologist for the consultation and having a longer consultation were both independently predictive of patient satisfaction of communication is discrepant with a recent finding that medical oncologists held longer consultations than did radiation oncologists [26]. This discrepancy may be due to the fact that the general systems history was excluded from analysis in the current study but not in the latter study, which also found that medical oncologists spent more time discussing history and symptoms than radiation oncologists.

The degree of patient-oncologist contact during the 12-week study interval may explain the finding that satisfaction with communication at 12 -weeks post-consultation was significantly greater for patients whose consultations were with a radiation rather than medical oncologist. Patients were likely

Table 5. Means and standard deviations for satisfaction with communication, global affective ratings, and MIPS consultation ratios

\begin{tabular}{lc}
\hline Variable & Mean (SD) \\
\hline Satisfaction with communication & \\
Post-consultation & $12.1(3.2)$ \\
I2-weeks post-consultation & $14.7(4.6)$ \\
Global affective ratings & \\
Patient & \\
Anxiety & $2.85(2.06)$ \\
Hostility & $0.07(0.30)$ \\
Assertiveness & $4.14(2.47)$ \\
Oncologist & \\
Patient centeredness & $5.81(2.36)$ \\
Friendliness & $6.77(1.99)$ \\
Sensitivity & $5.76(2.33)$ \\
Nervousness & $0.17(0.59)$ \\
MIPS consultation ratios & \\
Patient directedness & $0.76(0.75)$ \\
Patient centeredness & $11.0(12.7)$ \\
Psychosocial focus & $0.08(0.09)$ \\
\hline
\end{tabular}

to have met with their radiation oncologists weekly while receiving radiation therapy, while chemotherapy patients typically met with their medical oncologists only once or twice during the study interval (clinical associates and primary nurses tend to have more frequent contact with patients receiving chemotherapy than do medical oncologists). To the extent that patients had a positive relationship with their radiation oncologists, having more frequent contact with radiation oncologists during the study period may have facilitated positive patient ratings of satisfaction with communication. Another contributing factor to the finding of greater 12-week post-consultation satisfaction among patients whose primary adjuvant treatment consultations were with radiation oncologists is the fact that radiation therapy was more likely to have finished prior to the end of the 12-week study period compared with chemotherapy that frequently continued beyond this assessment point. It is therefore possible that the satisfaction ratings of chemotherapy patients may have been more heavily influenced by debilitating treatment side effects experienced at the time of the assessment.

Given the high overall patient satisfaction levels observed in this study, the significant predictors in this study are generally differentiating 'satisfied' patients from 'very satisfied' patients. This does not diminish the merit of the findings; evidence suggests that patients may report high satisfaction with consultations despite reporting unmet needs following these consultations [29]. Patient satisfaction scores have been shown to be clinically relevant in being reliably correlated with patient age and psychological well-being [16].

The findings of this study should be reviewed solely in the context of women with breast cancer; studies using patients with mixed genders and cancer diagnoses may yield different results. For example, a study of question asking by a diverse group of oncology patients - but including only two women with breast cancer-and their

Table 6. Breast sample statistically significant regression findings

\begin{tabular}{|c|c|c|c|c|}
\hline & $R$-square & $F$-value & df & $P$-value \\
\hline \multicolumn{5}{|l|}{ Post-consultation } \\
\hline Satisfaction with communication & 0.386 & 3.31 & 23,144 & $<0.001$ \\
\hline Age & & 6.23 & |144 & 0.014 \\
\hline Tumor size & & 6.81 & 1144 & 0.010 \\
\hline Consultation duration & & 11.25 & 1144 & 0.001 \\
\hline Oncologist specialty & & 6.09 & 1144 & $0.059^{*}$ \\
\hline Received consultation recording & & 2.82 & 1144 & $0.096^{*}$ \\
\hline \multicolumn{5}{|l|}{ I2-week post-consultation } \\
\hline Satisfaction with communication & 0.279 & 2.03 & 23,144 & 0.007 \\
\hline Tumor size & & 6.31 & | 144 & 0.013 \\
\hline Patient assertiveness (global rating) & & 5.34 & 1144 & 0.023 \\
\hline Oncologist specialty & & 4.11 & 1144 & 0.045 \\
\hline
\end{tabular}

*Marginal (trend) significance. 
companions found that, unlike the present study, the companions asked significantly more questions than patients [30]. In another study consisting of patients diagnosed with cancers of 1 of 11 primary sites (and patients with benign tumors and unknown primaries), clinician specialty (medical/ radiation/surgeon) was not significantly related to patient satisfaction [16].

The extraction of meaning from the information or 'message' conveyed during face-to-face communication is based on several factors, including the words of the message, the tone of voice, and body language. A limitation of present study is that the content analysis was performed on audiotapes rather than videotapes; audiotapes do not allow for analysis of non-verbal body language cues that may affect well-being. Audiotapes, however, are advantageous in being less invasive than videotapes; oncologists and patients are more receptive to being recorded using audiotape. The MIPS scoring system, like most other communication analytic schemes, is limited in being able to only classify instances of the spoken word or quantify the affective features of the consultation. The MIPS is not designed to account for 'silent messages' - the explicit or implicit messages conveyed by one party to another via responses of silence-that may impair effective consultation communication $[4,5]$.

A potential limitation of the MIPS scoring method is that, in an effort to explicate the consultation, the overall emotional tone or pulse of the consult may be lost in the measurement categories, despite the fact that the method allows for the scoring of patient cues and concerns and oncologist responses to these cues/concerns. Fortunately, the MIPS scoring method avoids this limitation with the inclusion of supplemental affective rating scales for the patient and oncologist. In this study, the observer ratings of patient affect/verbal behavior were significantly predictive of patient satisfaction with communication at 12-weeks post-consultation, while objective indices of patient-centered communication derived from the audiotape recordings were not significant. Patients who were rated as more highly assertive were less likely to be satisfied with communication. The reason for this finding is unclear but it is hypothesized that patients who are more vocal and ask more questions during the consultation do so because they are more concerned or upset about their disease course and/or oncology service delivery. This explanation is consistent with an earlier finding that patient satisfaction at 3-months post-consultation was significantly predicted by patient negative talk, patient directions, and patient anger [12]. This finding suggests that observers of patients' affect and verbal behavior during consultations may be able to accurately predict, based on their observations of affective qualities of speech, those patients who are at the greatest risk for dissatisfaction with communication.

The goal of the present study was not to compare the quality of communication across oncologists (although this is a worthy purpose) but rather to identify significant aspects of patient-oncologist communication during the primary treatment consultation that are suggestive of poor satisfaction with communication, with an aim to identify possible intervention targets for enhancing this outcome. The present study is a beginning step in this regard. Additional research is needed to identify the prime determinants of patient satisfaction with communication from the plethora of biomedical and psychosocial consultation factors, and considering the diverse mix of health professionals who communicate with cancer patients in the course of care delivery and thereby influencing patients' levels of satisfaction with communication. The current findings suggest that global, subjective ratings of patient affect, and the qualitative nature of patient verbal behavior are the important complement to objective measurement of consultation factors when predicting patient satisfaction with communication.

Future research in this area should be guided by theoretical models of communication in oncology, taking into consideration the goals that patients bring to the consultation, and the unmet communication needs that patients have along the cancer trajectory. While the present study has demonstrated that consultation factors are predictive of satisfaction with communication, future studies may benefit from moving beyond factors inherent to the consultation to consider aspects of the external environment as predictors of communication. For example, dissatisfaction with length of wait time in clinic prior to a consultation is a significant predictor of dissatisfaction with communication [16]. Future efforts require prospective, longitudinal designs with large samples to determine the patient, physician and contextual factors that shape patient satisfaction with communication from the time of diagnosis through the post-treatment period [31].

\section{Acknowledgements}

Supported by research grant $\sharp 013311$ from the Canadian Breast Cancer Research Alliance and research team grant $\sharp 010283$ from the National Cancer Institute of Canada (NCIC) with funds from the Canadian Cancer Society (CCS) and the CCS/NCIC Sociobehavioral Cancer Research Network. Thomas F. Hack is supported by a Dorothy J. Lamont Scientist Award from the NCIC and the Canadian Institutes of Health Research (CIHR). Lesley F. Degner is supported by a Research Chair from the Canadian Health Services and Research Foundation and the CIHR. 


\section{References}

1. Roter D. The enduring and evolving nature of the patient-physician relationship. Patient Educ Couns 2000;39:5-15.

2. Hall J, Roter DL, Katz NR. Meta-analysis correlates of provider behavior in medical encounters. Med Care 1998;26:657-675.

3. Stewart M, Brown JB, Weston WW, McWhinney IR, McWilliam CL, Freeman TR. Patient-Centered Medicine: Transforming the Clinical Method. Sage: Thousand Oaks, CA, 1995.

4. Brown RF, Bylund CL. Communication skills training: describing a new conceptual model. Acad Med 2008;83:37-44.

5. Feldman-Stewart D, Brundage MD, Tishelman C and the SCRN communication team. A conceptual framework for patient-professional communication: an application to the cancer context. Psycho-Oncol 2005; 14:801-809.

6. Ong LML, de Haes JCJM, Hoos AM, Lammes FB. Doctor-patient communication: a review of the literature. Soc Sci Med 1995;40:903-918.

7. Hack TF, Degner LF, Parker PA and the SCRN communication team. The communication goals and needs of cancer patients: a review. Psycho-Oncol 2005; 14:831-845.

8. Bredart A, Bouleuc C, Dolbeault S. Doctor-patient communication and satisfaction with care in oncology. Curr Opin Oncol 2005;17:351-354.

9. Butow PN, Dunn SM, Tattersall MH, Jones QJ. Computer-based interaction analysis of the cancer consultation. Br J Cancer 1995;71:1115-1121.

10. Uitterhoeve R, Bensing J, Dilven E, Donders R, deMulder P. Nurse-patient communication in cancer care: does responding to patient's cues predict patient satisfaction with communication. Psycho-Oncol 2009. Epub ahead of print.

11. Roberts CS, Cox CE, Reintgen DS, Baile WF, Gibertini M. Influence of physician communication on newly diagnosed breast patients' psychologic adjustment and decision-making. Cancer 1994;74(1 Suppl):336-341.

12. Ong LML, Visser MRM, Lammes FB, de Haes JCJM. Doctor-patient communication and cancer patients' quality of life and satisfaction. Patient Educ Couns 2000;41:145-156.

13. Lerman C, Daly M, Walsh WP, Resch N, Seay J, Barsevic A et al. Communication between patients with breast cancer and health care providers. Determinants and implications. Cancer 1993;72:2612-2620.

14. Zachariae R, Pedersen CG, Jensen AB, Ehrnrooth E, Rossen PB, von der Maase H. Association of perceived physician communication style with patient satisfaction, distress, cancer-related self-efficacy, and perceived control over the disease. Br J Cancer 2003;88:658-665.

15. Urden LD. Patient satisfaction measurement: current issues and implications. Lippincotts Case Manag 2002;7:194-200.

16. Shilling V, Jenkins V, Fallowfield L. Factors affecting patient and clinician satisfaction with the clinical consultation: can communication skills training for clinicians improve satisfaction? Psycho-Oncol 2003;12:599-611.

17. Hack TF, Pickles T, Bultz BD, Ruether JD, Weir LM, Degner LF et al. Impact of providing audiotapes of primary adjuvant treatment consultations to women with breast cancer: a multi-site, randomized, controlled trial. J Clin Oncol 2003;21:4138-4144.

18. Ford S, Hall A, Ratcliffe D, Fallowfield L. The medical interaction process system (MIPS): an instrument for analysing interviews of oncologists and patients with cancer. Soc Sci Med 2000;50:553-566.

19. Ford S, Fallowfield L, Lewis S. Doctor-patient interactions in oncology. Soc Sci Med 1996;42:1511-1519.

20. Fallowfield L, Lipkin M, Hall A. Teaching senior oncologists communication skills: results from phase 1 of a comprehensive longitudinal program in the United Kingdom. J Clin Oncol 1998;16:1961-1968.

21. Stewart M. Brown JB, Donner A, McWhinney I, Oates J, Weston WW. Impact of patient-centered care on patient outcomes in family practice: Final report. Thames Valley Family Practice Research Unit, London, Ont, 1996.

22. Henbest R, Stewart M. Patient-centredness in the consultation. 2: does it really make a difference? Fam Pract 1990;7:28-33.

23. Stewart M, Belle Brown J, Donner A, McWhinney IR, Oates J, Weston WW, Jordan J. The impact of patientcentered care on outcomes. J Fam Pract 2000;49: 796-804.

24. Stewart M, Belle Brown J, Hammerton $\mathrm{J}$ et al. Improving communication between doctors and breast cancer patients. Ann Fam Med 2007;5:387-394.

25. Epstein RM, Franks P, Fiscella K et al. Measuring patient-centered communication in patient-physician consultations: theoretical and practical issues. Soc Sci Med 2005;61:1516-1528.

26. Dimoska A, Butow PN, Dent E, Arnold B, Brown RF, Tattersall MHN. An examination of the initial cancer consultation of medical and radiation oncologists using the Cancode interaction analysis system. $\mathrm{Br} \mathrm{J}$ Cancer 2008;98:1508-1514.

27. Fagerlind H, Lindblad AK, Bergstrom I et al. Patientphysician communication during oncology consultations. Psycho-Oncol 2008;17:975-985.

28. Gattelllari M, Butow PN, Tattersall MH. Informed consent: what did the doctor say? Lancet 1999;353:1713.

29. Brown RF, Hill C, Burant CJ, Siminoff LA. Satisfaction of early breast cancer patients with discussions during initial oncology consultations with a medical oncologist. Psycho-Oncol 2009;18:42-49.

30. Eggly S, Penner LA, Greene M, Harper FWK, Ruckdeschel JC, Albrecht TL. Information seeking during bad news oncology interactions: question asking by patients and their companions. Soc Sci Med 2006;63(11):2974-2985.

31. Arora NK. Interacting with cancer patients: the significance of physicians' communication behavior. Soc Sci Med 2003;57:791-806. 